\title{
The spectrum of abdominal wall desmoid fibromatosis and the outcomes of its surgical treatment
}

\author{
I Bombil, ${ }^{(10}$ L Ngobese' ${ }^{(1)}$ \\ ${ }^{1}$ Department of Surgery, Chris Hani Baragwanath Academic Hospital, South Africa \\ ${ }^{2}$ Department of Anatomical pathology, University of the Witwatersrand, South Africa
}

Corresponding author, email: ifongobombil@gmail.com

\begin{abstract}
Background: Desmoid fibromatosis (DF) arises in musculoaponeurotic structures, most commonly the rectus abdominis muscle. It is locally aggressive with no propensity for metastasis. The aim was to describe the spectrum of abdominal wall DF and its surgical treatment and outcome at a tertiary institution.

Methods: All abdominal wall DF resected from 2007 to 2019 were retrospectively analysed. The tumours had a fullthickness abdominal wall excision. The defect was reconstructed with biological implants covered by either skin edge undermining or split skin graft. Histological diagnosis was based on characteristic morphological and immunohistochemistry parameters. Parameters analysed were demographics, lesion size, procedure performed and outcomes.

Results: Eleven female patients with a median age of 29 years (range 21-61) presented with rapid growth over 6-24 months of anterior abdominal wall tumours with a median maximum resected diameter of $163 \mathrm{~mm}$ (range 63-200 mm). There was no specific complication related to the abdominal wall reconstruction. With a median follow-up of 5 years (range 1-11 years), all patients displayed an asymptomatic abdominal wall bulge (eventration). One patient has had a small recurrence currently being observed.

Conclusion: Striking features of DF tumours at Chris Hani Baragwanath Academic Hospital (CHBAH) were their location, rapid growth, female gender and large size. Biological implant without complex plastic surgery techniques result in good outcomes, though the rate of progression of eventration to hernia is unknown.

Keywords: desmoid fibromatosis, abdominal wall, biological implants
\end{abstract}

\section{Introduction}

Desmoid fibromatosis (DF) is one of the rare (5-6 cases/ million) malignancies of mesenchymal origin arising from musculoaponeurotic structures throughout the body but occurs most commonly in the rectus muscle of the anterior abdominal wall..$^{1,2}$ It is a neoplastic proliferation of myofibroblast that often displays aggressive infiltrative growth with a tendency toward local recurrence but no propensity to metastasise. ${ }^{3}$

More than $80 \%$ of DF tumours are sporadic and in a small proportion $(5-10 \%)$ of cases they are linked to familial adenomatosis polyposis (FAP) and Gardner syndrome. ${ }^{4}$ Sporadic tumours harbour somatic mutations in $\beta$-catenin gene (CTNNB1), while FAP-associated tumours display germline mutations in adenomatosis polyposis coli (APC) gene. ${ }^{1,5}$ Both mutations though mutually exclusive in DF result in stabilisation and translocation of B-catenin to tumour cell nuclei as demonstrated by $\beta$-catenin immunohistochemistry nuclear positivity. 3,5 The mutated $\beta$-catenin is resistant to degradation and its accumulation acts as an oncoprotein.

The epidemiology of DF tumours is variable and displays a range of presentation in different age groups. In children, there is equal incidence in males and females with tumours frequently located at extra-abdominal sites. In young adults, there is a trend toward female predominance and the usual site is the abdominal wall. In adults over 40 years and the elderly, there is equal gender distribution and tumours are seen equally at abdominal and extraabdominal sites. ${ }^{6}$ Abdominal DF tumours typically arise in young females of childbearing age and often occur during gestation or following childbirth. ${ }^{6}$ The presentation is either asymptomatic (incidentally found during imaging) or a slow growing, painless, deep-seated mass. In rare cases, patients present with a rapidly expanding mass which may reach a large size. ${ }^{1,3}$

There are few publications on DF tumours in South Africa and around the world. ${ }^{7-10}$

An international multi-institution study in seven European sarcoma reference centres reported on the beneficial role of low dose chemotherapy in FAP-related desmoid tumour. ${ }^{11}$ Despite radical excision, DF tumours are prone to recur "Desmoid tumour don't die"10 - and radiotherapy has been used to reduce the risk of recurrence. Locally, Sharma et al. at the Johannesburg General Hospital reported in 2006 that the mainstay of treatment is surgery with no obvious benefit from radiotherapy. ${ }^{12}$ Pickard et al. from Groote Schuur Hospital reported similar results in 2019, although they emphasised the role of primary radiotherapy on patients whose tumour was unresectable as it produced a partial response or stabilisation of the disease. ${ }^{13}$ 
In this report, we reviewed patients with DF tumour treated at CHBAH and detail the clinical spectrum and present the results of surgical treatment.

\section{Method}

A retrospective analysis of 11 cases of DF tumours resected from January 2007 to December 2019 at CHBAH. Data were obtained from the theatre registry and patient files from the department of records. Parameters analysed were patient demographics, histological reports, the description of the procedure performed and outcome. Patients were treated with full-thickness resection of the anterior abdominal wall tumour from skin to peritoneum aiming for a clear radial margin of $2 \mathrm{~cm}$ and a complete resection (R0). Technical details of tumour resection and reconstruction method complications were recorded.

\section{Results}

Thirteen procedures were performed on 11 patients. All were female with a median of 29 years (range $21-61$ ). The excision of the abdominal wall and reconstruction were performed in all patients. The patients' characteristics and surgical management are depicted in Table I. There was no enterocutaneous fistula and all split skin grafts (SSG) were successful. Seven patients were available for follow-up with a median of 5 years (1-11 years) and revealed gradual weakening of the abdominal wall that was less pronounced in the group where Permacol ${ }^{\mathrm{TM}}$ and normal skin cover were used. No patient received adjuvant therapy.

\section{Discussion}

This study is in keeping with the literature where abdominal wall location is the most common tumour site in young adults. ${ }^{6}$ Although all patients are female, relationship with pregnancy was established in only one case where the tumour was noted a few months post-delivery. Another unusual finding in this study is the relatively rapid tumour growth (6-24 months) with nearly all patients presenting with very large tumours.

The international guidelines from the desmoid tumour working group recognise the role of low dose chemotherapy, radiotherapy and certain drugs (nonsteroidal antiinflammatory drugs (NSAIDs) and anti-oestrogen.,11,12 The antihormonal alone or in combination with NSAIDs reported partial, stable disease and disease progression of $25 \%, 65 \%$ and $10 \%$ respectively. Tyrosine kinase inhibitors were also tried in selected patients with unresectable disease. The overall response rate was $6 \%$. More than a third of patients required dose reduction whereas $10 \%$ discontinued the treatment due to side effects. The result from the international, multi-institutional retrospective analysis reported $54.1 \%$ of partial response, $40.55 \%$ of stable disease and disease progression in $5.4 \%$ of 37 patients treated from 2000 to 2018 with low dose methotrexate and vinca alkaloid (vinorelbine and or vinblastine). ${ }^{11}$

Although these therapies can be used on DF tumours at all locations, they are generally used in the context of advanced or rapidly progressive disease that is irresectable or would involve multi-organ resection. The recommendation from the joint global consensus-based guidelines approach for

\begin{tabular}{|c|c|c|c|c|c|c|}
\hline $\begin{array}{l}\text { Age } \\
\text { (years) }\end{array}$ & Size (mm) & $\begin{array}{c}\text { Lead time } \\
\text { (months) }\end{array}$ & Site & $\begin{array}{c}\text { Type of } \\
\text { biological } \\
\text { implant }\end{array}$ & Skin cover & Additional procedures \\
\hline
\end{tabular}

\begin{tabular}{lcccccc}
\hline \multicolumn{5}{c}{ Complex procedure: full-thickness abdominal wall excision + reconstruction with implant (inlay) + additional procedures } \\
+skin cover
\end{tabular}

* - recurrence, $\#$ - re-excision for involved margin, ** - tumour noted few months after delivery

RUQ - right upper quadrant, RLQ - right lower quadrant, LUQ - left upper quadrant, LLQ - left lower quadrant, SS - undermined surrounding skin, SSG - split skin graft, ICD - intercostal drain 
adult and paediatric patients emphasised the role of active surveillance in certain cases, relegating surgery or medical treatment to second-line therapy. When the disease progressed during active surveillance, preference was given to surgery for abdominal wall desmoid tumour or to medical therapy for intra-abdominal and extremities tumour. ${ }^{1,9}$

In comparing surgery alone versus surgery combined with radiation, the feeling was that radiation did not contribute much but was fraught with the risk of radiation-induced sarcoma.

In this study, we used biological implants as they are the safest option to place directly onto the bowel, and this is believed to provide regenerative repair. ${ }^{14-16}$ The nature of the biological implants makes them prone to biodegradability by host enzymatic activities (collagenase, protease). This can lead to loss of integrity of the implant and eventual abdominal wall eventration. Certain biologics (like Permacol $^{\mathrm{TM}}$ ) are cross-linked and bioengineered to resist the catalytic activity of the enzyme with expected satisfactory outcomes, considering the complexity of the abdominal wall defect. ${ }^{17}$ Both Strattice ${ }^{\mathrm{TM}}$ and XenMatrix ${ }^{\mathrm{TM}}$ are non-crosslinked acellular dermal reconstructive tissue matrix derived from porcine collagen. These are believed to provide durable abdominal wall support through their regenerative repair, biocompatibility and easy incorporation into the recipient tissue. However, that expectation was not met in our study as progressive abdominal wall weakness was noted. We did not come across any study that describes reconstruction of the abdominal wall after full-thickness excision except for a case report that shows no eventration after a short follow-up of 6 months, ${ }^{15,18}$ but the literature from the repair of incisional hernia with biological implant reports findings similar to our study when a biological implant is used as a bridge. ${ }^{14,17,19}$ Interestingly, no patient developed enterocutaneous fistula due to the presence of the biological implants. ${ }^{13,20}$

Nevertheless, we need to recognise that the biological implants alone are not expected to fulfil the role of satisfactory long-term abdominal wall substitute for full-thickness abdominal wall loss. We believe that such expectation is unrealistic. Biological implants are commendable in this study because they simplify the management of large abdominal wall defect that would otherwise have required complex plastic surgery reconstruction that is not without significant morbidity. Complex plastic reconstruction techniques are inappropriate to use as the primary procedure due to the possibility of local recurrence and donor site disability.

\section{Conclusion}

All our DF patients were female and had anterior abdominal wall tumours that had full-thickness abdominal wall excision. The resultant full-thickness defect was bridged with biological implants with minimal periprocedural morbidity. The recurrence rate was low in the $60 \%$ in whom follow-up was obtained. The abdominal wall-bridged defect exhibited gradual eventration with time, and it is unclear if further, more complex reconstructive abdominal surgery will be required for these patients.

\section{Conflict of interest}

The authors declare no conflict of interest.

\section{Funding source}

None.

\section{Ethical approval}

Ethical approval was obtained from the human ethics committee of the university of the Witwatersrand and the research review board of CHBAH (M191119).

\section{ORCID}

I Bombil (iD https://orcid.org/0000-0002-4819-0785

L Ngobese (iD https://orcid.org/0000-0001-8379-793X

\section{REFERENCES}

1. Desmoid tumour working group. The management of desmoid tumours - a joint global consensus-based guideline approach for adult and paediatric patients. Eur J Cancer. 2020;127:96107. https://doi.org/10.1016/j.ejca.2019.11.013.

2. Xiao J, Mao J, Li B. Clinical characteristic and treatment of intra-abdominal aggressive fibromatosis - a retrospective study of 16 patients. Front Med (Lausanne). 2020;7:2. https:// doi.org/10.3389/fmed.2020.00002.

3. Fisher C, Thway K. Aggressive fibromatosis. Pathology. 2014;46(2):135-40

4. Huss S, Nehles J, Binot E, et al. $\beta$-Catenin (CTNNB1) mutations and clinicopathological features of mesenteric desmoid-type fibromatosis - CTNNB1 mutations of mesenteric desmoids. Histopathology. 2013;62(2):294-304.

5. Rossi S, Laurino L, Dei Tos AP. Desmoid-type fibromatosis: from morphology to molecular genetics. Diagn Histopathol. 2008;14(11):546-51.

6. Fletcher C, Bridge J, Hogendoorn P, Pancras C, Mertens F. WHO Classification of tumours of soft tissue and bone. 4th ed (IARC WHO Classification of Tumours). Lyon: IARC Press; 2013. p.72-6.

7. Kasper B. Desmoid tumours: a perfect example for making progress in treatment management through international collaboration. ESMO Open. 2019;4:e000636. https://doi. org/10.1136/esmopen-2019-000636.

8. Papazoglou A, Komporozos V. Diagnosis and treatment of sporadic and familial adenomatous polyposis (FAP) associated desmoid tumours: literature review. Hellenic J Surg. 2018;90:299-307. https://doi.org/10.1007/s13126-0180494-7.

9. Ganeshan DM, Amini B, Nikolaidis P, Assing M, Vikram R. Current update on desmoid fibromatosis. J Comput Assist Tomogr. 2019;43(1):29-38. https://doi.org/10.1097/ RCT.0000000000000790.

10. Sifumba S, Thomson SR, Madaree A. Desmoids don't die. SAMJ. 1993;83(7):536-7.

11. Napolitano A, Provenzano S, Colombo C, et al. Familial adenomatosis polyposis-related desmoid tumours treated with low-dose chemotherapy - results from an international, multiinstitutional, retrospective analysis. ESMO Open. 2020;5(1). https://doi.org/10.1136/esmoopen-2019-000604.

12. Sharma V, Chetty DN, Donde B, et al. Aggressive fibromatosis - impact of prognostic variables on management. S Afr J Surg. 2006;44(1):6-8, 10-11.

13. Pickard HDP, Jacob N, Malherbe F, et al. The management of desmoid tumours at Groote Schuur Hospital - a retrospective review of current practice. South African Journal of Oncology. 2019;3. https://doi.org/10.4102/sajo.v3i0.68.

14. Patel KM, Nahabedian MY, Albino F, Bhanot P. The use of porcine acellular dermal matrix in a bridge technique for complex abdominal wall reconstruction - an outcome analysis. Am J Surg. 2013;205(2):209-12. https://doi.org/10.1016/j. amjsurg.2012.05.031. 
15. Hackenberger PN, Poteet SJ, Janis JE. Bridging acellular dermal matrix in abdominal wall repair following radical resection of recurrent endometrioma. Plast Reconstr Surg Glob Open. 2020;8(1):e2603. https://doi.org/10.1097/ GOX.0000000000002603.

16. De Vries FEE, Hodgkinson JD, Claessen JJM, et al. Longterm outcomes after contaminated complex abdominal wall reconstruction. Hernia. 2020. https://doi.org/10.1007/s10029020-02124-7.

17. Russello D, Sofia M, Conti $\mathrm{P}$, et al. A retrospective, Italian multicentre study of complex abdominal wall defect repair with a Permacol biological mesh. Sci Rep. 2020;10(1):3367. https://doi.org/10.1038/s41598-020-60019-0.

18. Couto Netto SD, Teixeira Jr F, Menegozzo CAM, et al. Abdominal wall reconstruction after desmoid type fibromatosis radical resection - case series from a single institution and review of the literature. Int J Surg Case Rep. 2017;33:167-72. https://doi.org/10.1016/j.ijscr.2017.02.050.

19. Albino FP, Patel KM, Nahabedian MY, et al. Immediate, multistaged approach to infected mesh - outcomes after abdominal wall reconstruction with porcine acellular dermal matrix. Ann Plast Surg. 2015;75(6):629-33.

20. Zhao X, Cao Z, Nie Y, et al. Retrospective analysis of defect reconstruction after abdominal wall tumour resection in 30 patients. Hernia. 2021;25:375-81. https://doi.org/10.1007/ s10029-020-02219-1. 